\title{
Monitoring and Diagnosis System of Downhole Tubing Leakage
}

\author{
Yunpeng YANG ${ }^{\mathrm{a}, 1}$, Jianchun FAN ${ }^{\mathrm{b}}$, Di LIU ${ }^{\mathrm{a}, \mathrm{b}}$, Fanfan $\mathrm{MA}^{\mathrm{b}}$ \\ ${ }^{a}$ China University of Petroleum-Beijing, China \\ ${ }^{\mathrm{b}}$ The Third Research Institute of China Electronics Technology Group Corporation, \\ China
}

\begin{abstract}
The downhole tubing in a gas well is affected by many factors such as high pressure erosion, gas lift operation, sand production at the bottom of the well and engineering construction, etc., which can easily lead to leakage of the threaded joints of the tubing and the pipe body, and the leaked natural gas will invade Annulus, making the annulus under pressure. The annular pressure caused by oil pipe leakage is a major safety hazard in oil and gas production. Therefore, the accurate diagnosis the degree of leakage of downhole tubing is of great significance to preventing the occurrence of production accidents effectively. To this end, a set of downhole tubing leak monitoring and diagnosis system has been developed by integrating fluid monitoring, acoustic wave detection and tracer detection technology, and the developed tubing leak monitoring and diagnosis system was used for leak detection tests on offshore platforms. The test results show that the developed tubing leakage monitoring and diagnosis system can meet the need of offshore gas well diagnosis, and realize the holographic diagnosis of the leakage degree of the downhole tubing without moving the downhole tubing string.
\end{abstract}

Keywords. Gas well; tubing leakage; monitoring; diagnosis system; holographic diagnosis

\section{Introduction}

The pressure in annulus caused by tubing leakage continues to threaten the safe production of gas well. Active measures should be taken to diagnose the leakage status. At present, major domestic and foreign oil and gas group companies and related scholars mainly adopt two methods: downhole detection and wellhead detection. Downhole detection is a direct and effective detection method. Common downhole detection technologies include: temperature logging, noise logging, ultrasonic logging, electromagnetic logging, etc. These methods have a large number of application cases in logging, and excellent results have been achieved. But downhole testing technology requires the testing equipment to be placed in the downhole tubing or casing, the production well needs to be shut down, or even the downhole tubing string needs to be moved, which will inevitably bring huge economic losses and safety risks. Therefore, the development of tubing Leakage wellhead detection technology is very necessary, and it is also an important direction of scientific research for major oil and gas group companies and related universities. Research on tubing leakage wellhead detection

${ }^{1}$ Corresponding Author: Yunpeng Yang, China University of Petroleum, Beijing, China; E-mail yy1914624371@163.com 
technology as early as 1995, RJ Hwang et al. studied the use of changes in crude oil composition during different production periods to determine the leakage between different completion strings of oil wells. However, the implementation of this method requires years of experience. Based on crude oil analysis data, rapid detection is not possible [1]. With the in-depth study of the annulus pressure problem, new achievements have been made in the field of downhole leakage detection. In 2000, Adam T. Bourgoyne et al. proposed to sample annulus fluid and analyze its hydrocarbon composition when the annulus was depressurized, and then compared it with the composition of known pay zones to determine whether the annulus pressure was caused by leakage [2]. They also believe that the growth rate of annulus pressure is related to the size and location of downhole leaks. The API RP90 standard introduces a method for judging the general position of tubing leakage based on the shut-in pressure test: close the downhole safety valve (surface controlled subsurface safety valve, SCSSV), release the pressure on the upper part of SCSSV, if the pressure of A annulus drops, then the leakage point is located in the upper part of the SCSSV, and the leakage in the lower part of the SCSSV can be judged by lowering the bridge plug into any position in the tubing [3]. In 2012, Zhu H. et al. studied the mechanism and prediction model of pressure rise in the "A" annulus in $\mathrm{CO} 2$ injection wells, they proposed a method for predicting the location of tubing leaks based on the principle of pressure balance. They believed that when the annulus pressure reached a stable level, The internal and external pressures at the leak point are balanced, and the iterative calculation process of the leak point position is given [4]. Later, I. Tarmoom et al. proposed the measurement of pressure, flow, fluid composition and other parameters in the comprehensive annulus pressure relief process to estimate the annulus leakage rate, and developed a set of annulus pressure relief rate measurement system in Adma-Opco. The field measurement application [5]. The Norwegian ScanWell company has also developed a monitoring system that integrates the leak detection system (LMS), sonic logging system (AMS), tracer detection system (TMS), component analysis and other functions. Among them, LMS is used to measure downhole wells. The leakage rate of gas, liquid or gas-liquid two-phase at the barrier failure position. AMS is used to confirm abnormal conditions of tubing and annulus and to detect liquid level changes. TMS is used to confirm the leakage depth of tubing and casing, which can realize the Diagnose the leakage status of downhole tubing and casing under the condition of column. Fan Jianchun and his colleagues at the China University of Petroleum have also made significant contributions to the study of annulus pressure, especially in the identification of acoustic leakage, and have proposed a method for the location of downhole casing leakage based on acoustic leakage.

Based on the characteristics of gas wellbore structure and string leakage acoustic waves, a set of downhole tubing leak detection system is designed that integrates multifluid parameters, acoustic wave method and tracer detection. Among them, acoustic wave method is used to detect annulus fluid level and pipe leakage. The column leakage point is located, and the tracer method is used to estimate the equivalent size of the leakage hole. 


\section{The composition and principle of the system}

\subsection{The Composition of the system}

As shown in Figure 1: The wellbore leakage monitoring system is mainly composed of fluid monitoring and signal acquisition, liquid level detection, leak detection, and tracer detection systems.

- Fluid monitoring: Use pipelines to introduce the annular air into the system, and monitor the gas status during the depressurization process. The main monitoring parameters include the flow rate, pressure and temperature in the pressure relief pipeline, and at the same time sampling and analyzing its composition at the pressure relief port.

- Liquid level detection: Estimating the depth of the annulus liquid level by testing the liquid level echo signal.

- Leak point detection: According to the structure of the Christmas tree tubing four-way, the flow channel of the four-way gate valve is connected to the annulus, and the acoustic wave sensor can be placed in it. When the gate valve is opened, the acoustic wave in the annulus can be received signal.

- Tracer detection: It mainly includes tracer injection and detection. The tracer is injected from the annulus, and the tubing and adjacent annulus outlets are inspected, and the gas well production is not affected during the inspection process. Non-polluting helium gas is selected as the tracer source. Considering that the annulus pressure is generally high, the tracer needs to be pressurized before it can be injected successfully, and the gas produced needs to be depressurized before the test is performed. The whole process includes three modules: helium pressurization-gas injection-helium detection.

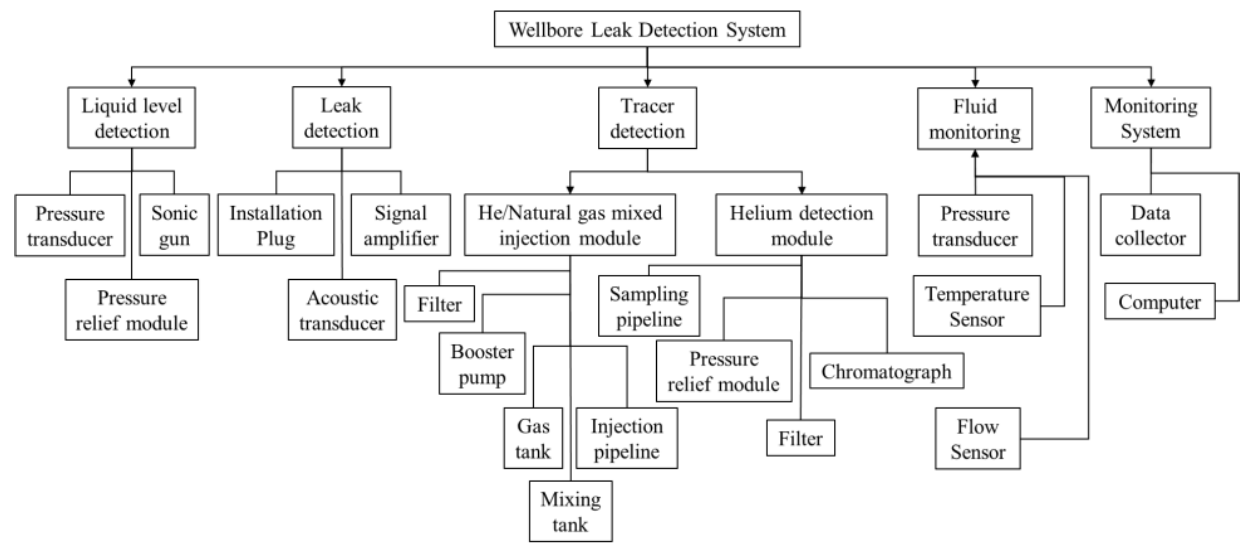

Figure 1. The composition of the downhole tubing leak monitoring and diagnosis system

\subsection{Principle}

According to APR RP90, the causes of pressure in the oil jacket annulus include thermal effects, gas lift residual pressure, oil pipe leakage and production casing leakage [3]. After confirming the existence of the leakage, it is necessary to determine the specific 
leakage source. As shown in Figure 2, a comprehensive diagnosis and analysis process for oil pipeline leakage is established based on logical reasoning:

(1) Leak identification

a. When the pressure $\mathrm{p} 1$ of annulus is greater than $100 \mathrm{psi}$ (approximately $0.69 \mathrm{MPa}$ ), it is considered that there is a possibility of leakage, and further detection and analysis are required.

b. Test the annulus liquid level L, then perform pressure relief, and define the pressure relief gradient $\Delta \mathrm{p}$. The liquid level test is performed every time the annular pressure drops by $\Delta \mathrm{p}$, and the dynamic liquid level $\mathrm{L}$ in the annular space during the pressure relief process is obtained.

c. When the annulus pressure drops by a certain value, the pressure relief is stopped, and the annulus sound wave signal is detected. Extract the characteristic value of the acoustic signal (RMS, crest factor, form factor, peak-to-peak value, root square amplitude, etc.) and compare it with the unpressurized (No leakage condition) annulus acoustic signal to determine whether there is leakage downhole.

d. When a downhole leak is identified, perform helium tracer detection. If the helium is detected at the outlet of the tubing, there is a leak in the tubing; if helium is not detected at the outlet of the tubing, the production casing leaks, and the pressure in the annulus of the tubing is caused by the intrusion of the outer annulus fluid.

e. The pressure change in the outer casing is monitored during the pressure relief of the oil casing annulus. If the outer annulus pressure $\mathrm{p} 2$ changes, there is leakage in the production casing; on the contrary, if there is no significant change in $\mathrm{p} 2$, there is no leakage in the production casing.

f. If there is no change in $\mathrm{p} 2$ and a downhole leak is identified or helium gas at the outlet of the tubing is detected, it means that only the tubing has a leak. If $\mathrm{p} 2$ changes significantly, and downhole leakage or helium return from the tubing outlet is identified, it indicates that both tubing and production casing are leaking. At this time, the production fluid will enter the oil casing annulus and technical casing annulus through the leak hole in turn., Causing the pressure of the outer annulus to rise sequentially. This situation poses the greatest threat to the safe production of offshore gas wells. Once this situation is discovered, it is necessary to immediately formulate strict control measures to prevent the pressure from further breaking through the outer annulus barrier and causing irreparable losses.

(2) Leak location and quantitative diagnosis process

a. When it is recognized that there is a leaking sound wave in the annulus of the oil jacket, autocorrelation processing is performed on the leaking sound wave signal, the characteristic time of the leaking sound wave signal is extracted, and the annular sound velocity distribution curve is combined to estimate the oil casing leak position $Z 1$.

b. Similarly, when a leak is identified in the outer annulus, sound wave detection is performed to estimate the location of the leak point Z2.

c. Further, the tracer test proves that when only the tubing leaks, the leaking position of the tubing is, using the liquid level $\mathrm{L}$ in the annulus of the oil casing, the leak point depth $\mathrm{Z} 1$, and the tracer concentration $\mathrm{Ct}$ at the tubing outlet, combined with the wellbore and production Information, estimate the equivalent hole diameter $\mathrm{d}$ of the oil pipe leakage.

$\mathrm{d}$. When it is recognized that the tubing and the production casing are leaking at the same time, the leakage point of the tubing at this time is $(\mathrm{Z} 1-\mathrm{Z} 1 \cap \mathrm{Z} 2)$, that is, the leakage point on the production casing is eliminated first, and then the tubing leakage is quantitatively analyzed. 
The diagnosis of the outer annulus follows the same process.

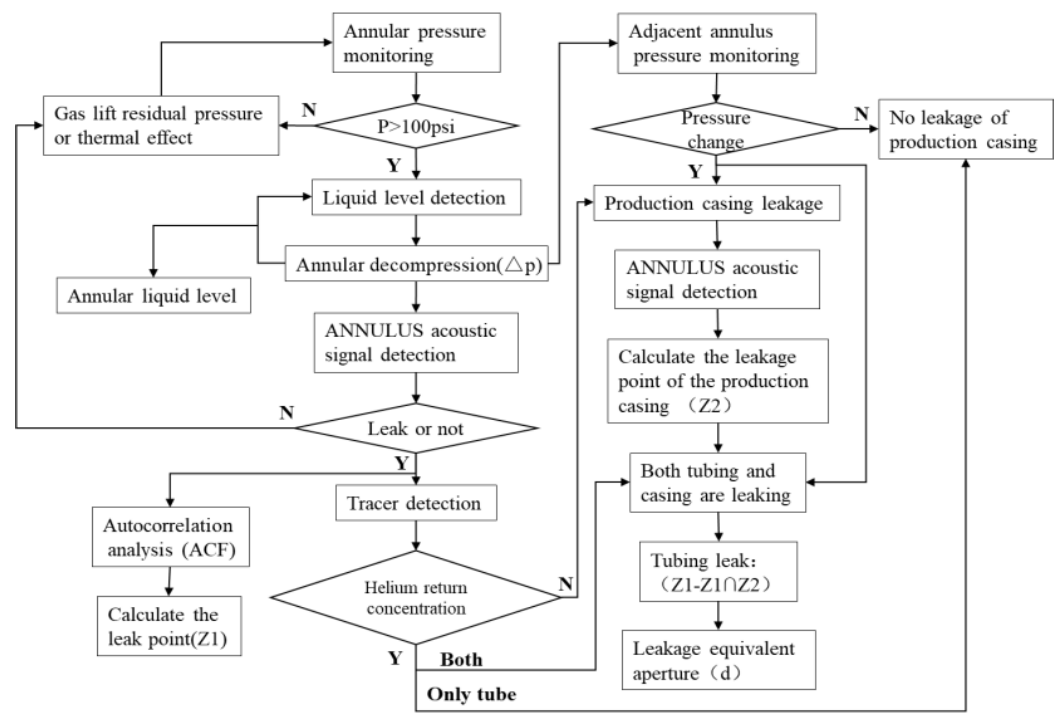

Figure 2. Comprehensive diagnosis process of oil casing leak

\subsection{Liquid Level Detection}

The principle of liquid level detection is based on the acoustic wave method, and the calculation formula is shown in formula (1). The key is to obtain the time $t$ for the sound wave to propagate from the wellhead to the liquid surface, and the propagation speed $\mathrm{v}$ of the sound wave in the annulus. The speed of sound $\mathrm{v}$ can be calculated using equation (2) [6].

$$
\begin{aligned}
& H=v t \\
& v=\left[\left(\frac{c_{p}}{c_{v}}\right)\left(\frac{R T_{g}}{M_{g}}\right)\left(Z_{g}+\rho\left(\frac{\partial Z_{g}}{\partial \rho}\right)_{T g}^{1 / 2}\right)\right]
\end{aligned}
$$

Where: $\mathrm{cp}$ and $\mathrm{cv}$ are the specific heat capacity at constant pressure and constant volume respectively, $\mathrm{J} /(\mathrm{mol} \cdot \mathrm{K}) ; \mathrm{R}$ is the general gas constant $\mathrm{J} /(\mathrm{mol} \cdot \mathrm{K}) ; \mathrm{Tg}$ is the temperature, $\mathrm{K} ; \mathrm{Zg}$ is the compressibility factor; $\rho$ is the mole Density, $\mathrm{mol} / \mathrm{m} 3$; $\mathrm{Mg}$ is molar mass, $\mathrm{kg} / \mathrm{mol}$, and the medium is annular air. 


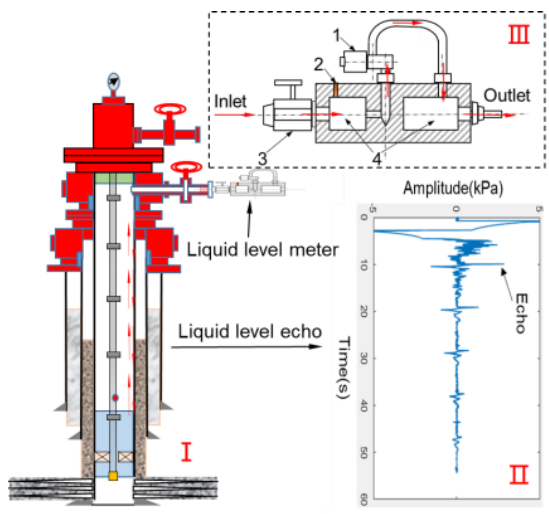

Figure 3. Principle of liquid level detection (1- Solenoid; 2- Acoustic transducer; 3- Ball valve; 4- Pressure chamber)

The sonic gun shown in Figure 3 (III) is used to launch a test wave into the annulus. When the test wave propagates to the bottom of the annulus, it will reflect on the liquid surface of the annulus. The liquid level echo signal is obtained at the wellhead of the annulus-Figure 3 (II) Determine the depth of the annulus liquid surface by extracting the echo time combined with the annulus sound velocity distribution law. Its working principle is: install it on the four-way gate valve of the Christmas tree tubing using a pipeline. After the ball valve is opened, there is a pressure difference between the two ends of the sonic gun. The high-pressure gas is released instantly by opening and closing the solenoid valve, and the high-pressure gas flows through the solenoid valve pressure fluctuations are generated during the channel and propagate into the annulus along the gas medium. The pressure fluctuations are the test waves in the liquid level test process.

\subsection{Principle of Leak Location}

When the oil pipe leaks, high-pressure natural gas enters the production annulus through the leak hole. As shown in Figure 4, a continuous acoustic signal is generated at the leak hole. Part of the acoustic signal propagates along the annulus to the wellhead, and the other part propagates downhole to the annulus liquid surface, and is transmitted to the wellhead after being reflected by the liquid surface. Install a sound wave receiving sensor at the exit of the Christmas tree tubing pipe to obtain the sound wave signal in the annulus. The acoustic signal collected at the wellhead in the annulus is the superposition of the leaked acoustic wave after different delays. The delay has the following relationship with the relative position of the leak in the annulus [7]:

$$
X=\bar{v}\left(t_{2}-t_{1}\right) / 2
$$

Where $\mathrm{x}$ is the distance between the leaking point and the reflecting surface of the tubing hanger; $\mathrm{t} 1$ is equal to the time of a return journey between the leaking sound wave from the leaking point to the annular liquid surface; $t 2$ is equal to the one between the leaking sound wave hanging from the tubing to the annular liquid surface Return time. 


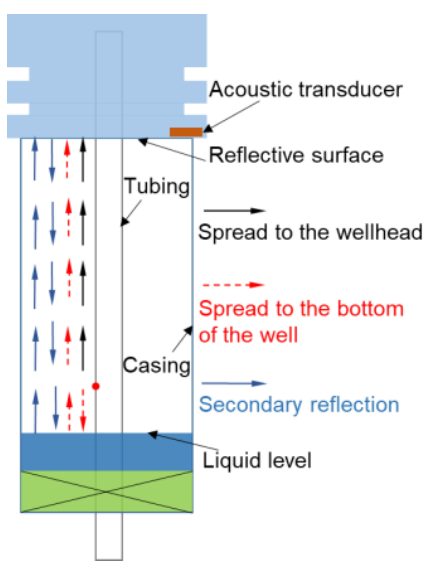

Figure 4. Schematic diagram of oil pipe leakage location

\subsection{Principles of Tracer Detection}

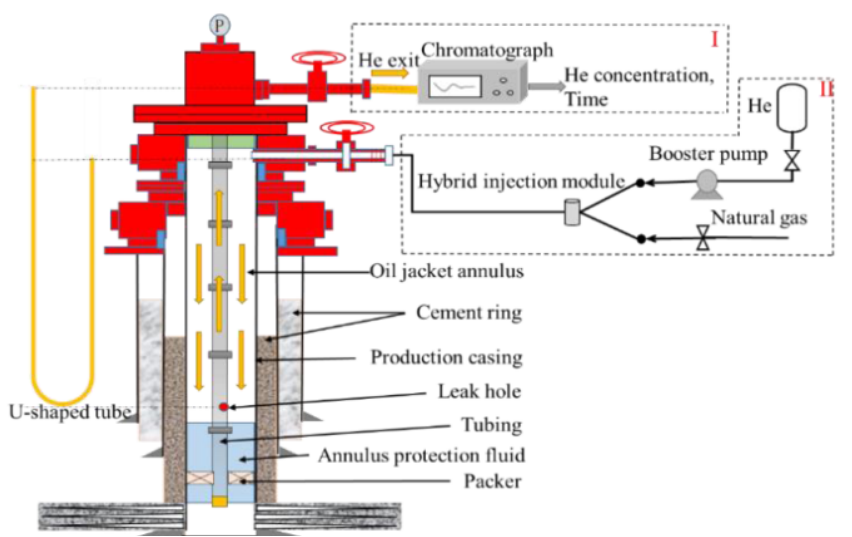

Figure 5. Schematic diagram of tracer detection

As shown in Figure 5, when the tubing leaks, a certain amount of tracer (helium is used as the tracer for leak detection in the text [8-10]) and natural gas mixture is continuously injected from the annulus wellhead. Driven by the air pressure, the mixed gas diffuses down the annulus to the leak point, passes through the leak point and enters the tubing, mixes with the natural gas in the tubing, and flows to the wellhead along with the produced gas. Collect and detect the concentration of helium in natural gas at the wellhead, and quantitatively analyze the leakage degree of the tubing according to production parameters, wellbore parameters and helium injection parameters. Assuming that the initial helium concentration is $\mathrm{C} 0$, the injection time is $\mathrm{t}$, the production parameter is $\mathrm{Y}$, the wellbore parameter is $\mathrm{B}$, the injection parameter is $\mathrm{X}$, and the leakage is $\mathrm{Q}$, the concentration of the returned helium can be expressed as:

$$
C=C_{o} f(t, B, X, Y, Q)
$$

The reverse solution can be obtained Oil pipe leakage [11]. 


\subsection{System}

Integrating fluid monitoring, liquid level detection, leak detection and tracer detection and other parameters, the downhole tubing and casing leak diagnosis system developed is shown in Figure 6. Its working principle is shown in Figure 7, and the components are shown in Table 1. Show.

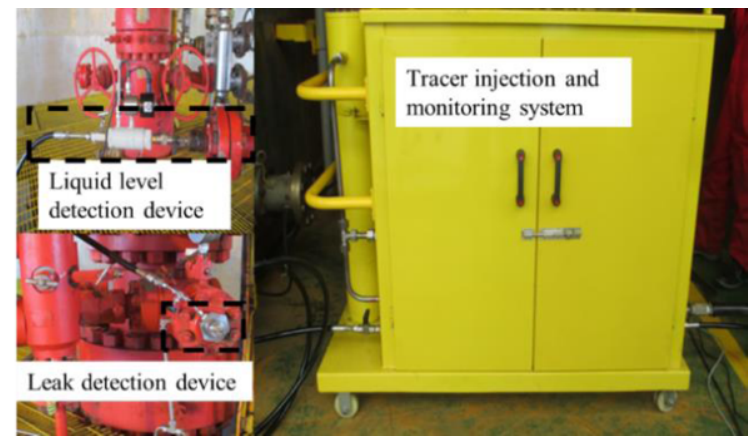

Figure 6. Physical map of downhole tubing and casing leak diagnosis system

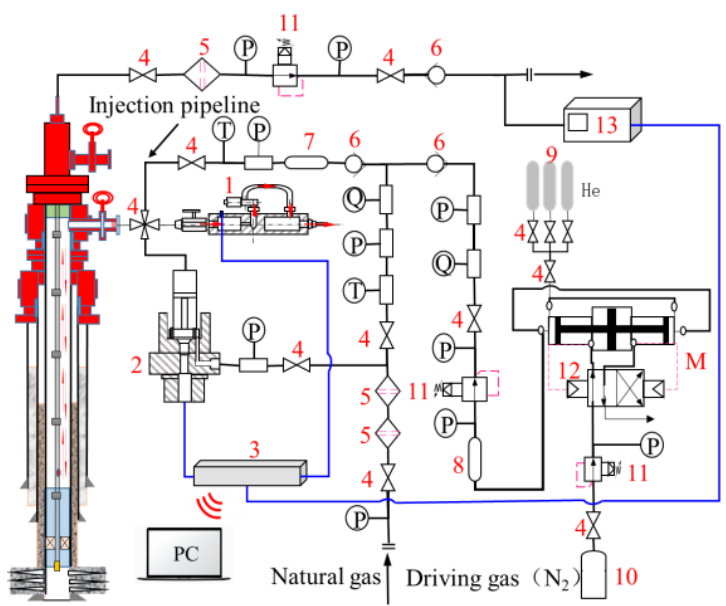

Figure 7. Working principle diagram of downhole casing leakage diagnosis system

Table 1. Summary of components of the downhole tubing and casing leak monitoring and diagnosis system

\begin{tabular}{ccccc}
\hline No. & Name & No. & Name \\
\hline 1 & Liquid level detection device & 8 & Gas Tank \\
2 & Leak point detection device & 9 & Helium cylinder \\
3 & surveillance system & 10 & Driving gas (N2) \\
4 & Valve & 11 & Pressure reducing valve \\
5 & Oil-gas separation device & 12 & Supercharger \\
6 & Check valve & 13 & Chromatograph \\
7 & Mixing tank & $\mathrm{P}$ & Pressure transmitter \\
$\mathrm{T}$ & Temperature transmitter & $\mathrm{Q}$ & Mass flowmeter \\
\hline
\end{tabular}

The detailed operation process is:

(1) Leak identification: Close the gate valve, drain the residual pressure on the outside of the gate valve, connect the liquid level detection device No. 1 shown in Figure 
7 to the wellhead four-way annulus outlet, and open the gate valve after completing the tightness test of each pipeline. Quickly open and close the solenoid valve and collect the annulus sound wave signal. After the test is over, close the gate valve, vent the residual pressure in the external pipeline, and then remove the liquid level detection device.

(2) Leak sound wave detection: Close the gate valve, drain the residual pressure on the outside of the gate valve, install the threaded plug with acoustic wave sensor number 2 as shown in Figure 7 at the wellhead four-way annulus outlet, open the gate valve slowly to perform annulus acoustic wave test. In order to avoid the moment when the gate valve is opened, the high-pressure gas in the annulus impacts and destroys the diaphragm of the acoustic wave sensor, the annular air body is reversely introduced through the pressure relief hole on the threaded plug, so that the pressure in the four-way annulus outlet is equal to or It is close to the pressure in the wellbore annulus, reducing the possibility of damage to the acoustic wave sensor after being impacted.

(3) Tracer test: Switch the downhole tubing and casing leak diagnosis system to the tracer injection module. When the tracer is injected, a gas source well with a pressure higher than the measured annulus pressure is required to drive the tracer to rapidly diffuse down the annulus to the leak hole. Injecting the mixture of tracer and natural gas in a slug manner can greatly reduce the amount of tracer. As shown in Figure 7, the tracer 9 is mixed with the high-pressure natural gas in the mixing tank 7 in a certain proportion through the pressurizing mechanism 12. The booster pump is driven by compressed nitrogen 10. Open the gate valve and the injection pipeline valve, inject the tracer into the annulus, and record the injection time, injection flow rate, injection temperature and pressure and other fluid parameters. After a period of injection, the injection of the tracer is interrupted, and the high-pressure natural gas is continuously injected, which drives the tracer to move quickly into the annulus. While injecting the tracer, open the sampling valve connected to the outlet of the tubing, and use the gas chromatograph number 13 shown in Figure 7 to continuously monitor the natural gas produced at the wellhead. Since the gas chromatograph sampling tube is an ordinary low-pressure equipment, the produced natural gas needs to be filtered and depressurized, and the pressure of the sample after depressurization is about $0.1 \mathrm{MPa}$. Considering that the endothermic reaction of natural gas in the process of pressure reduction and flow restriction is likely to cause ice blockage in the pressure relief pipeline, the system adopts a needle valvereducing valve combination multi-stage pressure reduction method. After the test is completed, close each valve in turn, and after evacuating the residual pressure in the system, remove the connected pipeline and restore the wellhead.

\section{Engineering Practice and Results}

Using the developed downhole tubing and casing leak monitoring and diagnosis system, 15 well-time detection experiments were carried out in the offshore annulus killing well. Taking the A5 detection result as an example, the test results are shown in Table 2, and the liquid level detection signal is shown in Figure 8, the time-domain diagram of the annulus acoustic signal and its autocorrelation curve are shown in Figure 9.

Table 2. Test results of Well A5

\begin{tabular}{rrrrrr}
\hline Well & Annulus & Pressure/MPa & \multicolumn{3}{c}{ Results } \\
\hline \multirow{2}{*}{ A5 } & \multirow{2}{*}{$\mathrm{A}$} & \multirow{2}{*}{9.14} & Liquid level $/ \mathrm{m}$ & Leak location $/ \mathrm{m}$ & Size $/ \mathrm{mm}$ \\
\cline { 4 - 6 } & & & 2070.71 & 853.3 & $2.5 \mathrm{~mm}$ \\
\hline
\end{tabular}




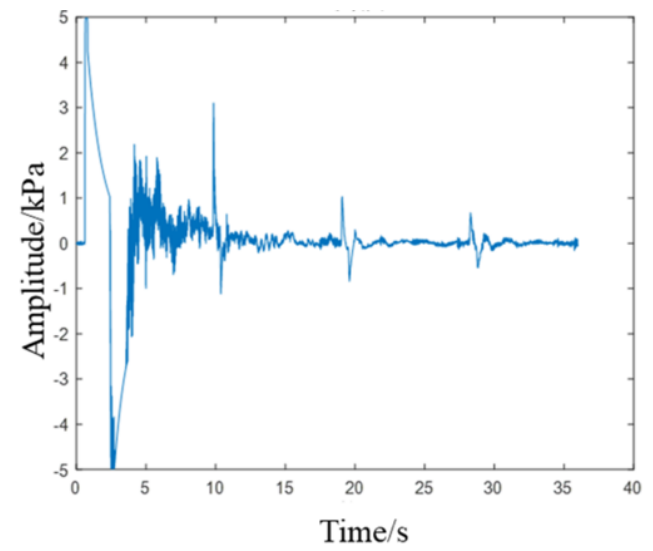

Figure 8. Annular liquid level test curve
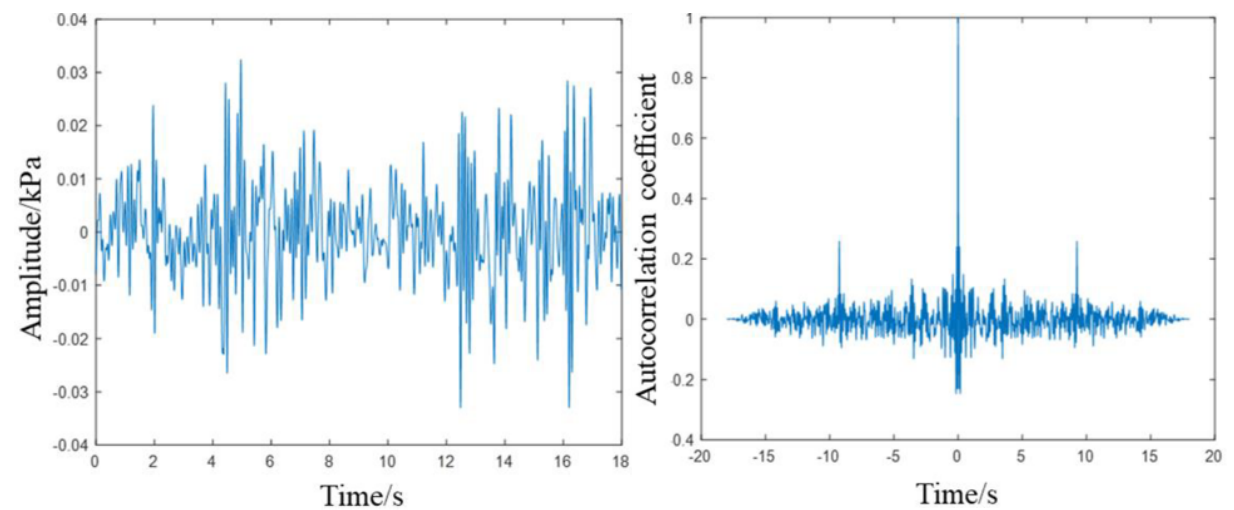

Figure 9. Time domain diagram of annulus acoustic signal and its autocorrelation curve

During the tracer test, continuously monitor the flow and pressure of the injected natural gas and the pressure in the wellhead annulus and tubing, and draw the change curve of each parameter as shown in Figure 10. A slug-type helium injection is used, the injection time is $137 \mathrm{~s}$, the slug length is $2.01 \mathrm{~m}$, and the flow of helium is zero after the injection is stopped. With the continuous injection of natural gas, the pressure in the annulus gradually increases, the injection flow rate gradually decreases, and the injection pressure and the annulus pressure are always consistent. With the continuous injection of natural gas, the injection pressure and the annulus pressure are slowly higher than the pressure in the tubing, and the pressures of the two stabilized at $13.13 \mathrm{MPa}$. At this time, the pressure in the tubing was $12.22 \mathrm{MPa}$. During this period, the gas chromatograph was used to continuously monitor the helium concentration in the tubing. Under this working condition, the peak return concentration of helium at the tubing outlet was about 177 ppm, and the peak time was $338 \mathrm{~min}$. The helium detection concentration is summarized as shown in Figure 11. Based on the pressure, flow, temperature and other parameters monitored by the tracer, combined with the detection results of the liquid level and the depth of the leak, the equivalent aperture of the leak is calculated to be $2.5 \mathrm{~mm}$. Using this equivalent aperture to calculate the theoretical concentration curve of helium returning in the forward direction is shown in Figure 11, which is more consistent with the detection concentration curve of helium. 


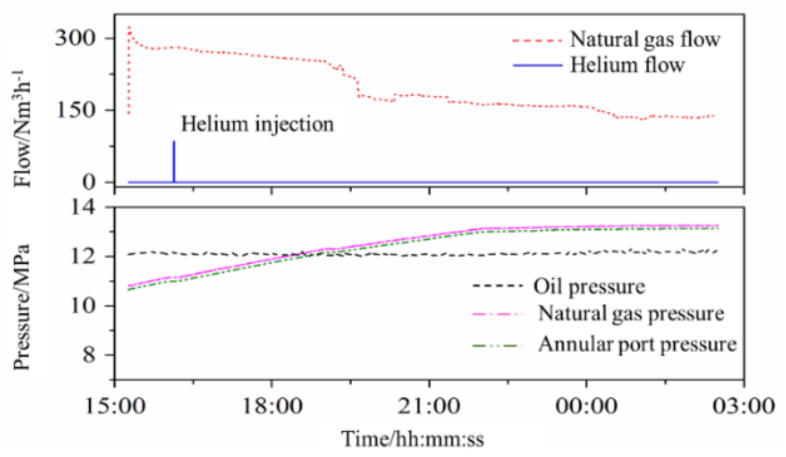

Figure 10. Variation curve of various parameters during the tracer test

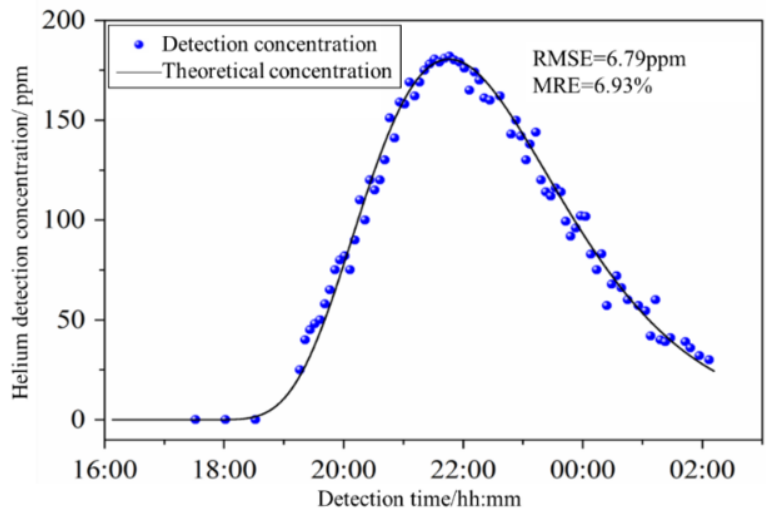

Figure 11. Comparison of theoretical concentration and detection concentration of helium

\section{Conclusion}

A single detection method cannot fully obtain downhole leakage information. The development of a gas well downhole tubing leakage monitoring and diagnosis system suitable for field use has always been the direction of field staff and scientific research workers.

(1) Integrating the existing fluid monitoring, liquid level detection, leak acoustic wave positioning and tracer detection technologies, a comprehensive diagnostic analysis process for tubing and casing leaks is established based on logical reasoning, in order to realize the downhole leak status (leak location, leak degree and the wellhead holographic diagnosis of the leak source) provides theoretical support.

(2) Integrated fluid monitoring, liquid level detection, leak acoustic wave positioning and tracer detection technologies, a downhole tubing and casing leak monitoring and diagnosis system was developed, and using this system, 15 detection experiments were carried out in the offshore annulus zone to kill wells. The data on the depth of the annulus liquid level, the depth of the downhole leak and the size of the leak are realized, and the holographic diagnosis of the downhole leakage state at the wellhead without moving the pipe string and affecting the production can be provided to ensure 
the safe production of gas wells. Effective technical support provides an important basis for the next comprehensive treatment of well killing in the annulus zone.

\section{References}

[1] Hwang R J, Elsinger R J. Detecting production tubing leak by time resolved geochemical analysis of oils[C]. The Production Operations Symposium, Oklahoma, U.S.A., 1995: 67-79.

[2] Bourgoyne A T, Scott S L, Regg J B. Sustained casing pressure in offshore producing Wells [C]. The 1999 Offshore Technology Conference, Houston, Texas, 1999.

[3] API RP90, Annular casing pressure management for offshore wells[S]. Washington: API, 2006.

[4] Zhu H, Lin Y, Zeng D, et al. Mechanism and prediction analysis of sustained casing pressure in "A" annulus of CO2 injection well [J]. Journal of Petroleum Science \& Engineering, 2012, 92-93:1-10.

[5] Tarmoom I, Thabet H B, Samad S, et al. A comprehensive approach to well-integrity management in Adma-Opco[C]. The 15th SPE Middle East Oil \& Gas Show and Conference, Manama, Bahrain, 2007.

[6] ASSOCIATION A G. AGA Report No. 10 - Speed of sound in natural gas and other related hydrocarbon gases[R]. Transmission Measurement Committee, 2002: 12-17.

[7] Liu D, Fan J C, Wu S N. Acoustic Wave-Based Method of Locating Tubing Leakage for Offshore Gas Wells. Energies, 2018, 11(12): 3454.

[8] Kohl J, Newacheck R L, Anderson E E. Locating casing shoe leaks with radioactive argon[J]. Petroleum Transactions, AIME, 1995, 204: 213-216.

[9] Shnaib F, Nadar M S, Sreekumar M P, et al. Successful application of CO2 tracer technology for surveillance of gas lifted wells[C]. The SPE Production and Operations Conference and Exhibition, Tunis, Tunisia, 2010.

[10] Li F. Research and Application of Gas Tracer Monitoring Technology[D]. Dongying: China University of Petroleum (East China),2013.

[11] Liu D. Resraech on Ground Detection and Diagnosis Method for Tubing and Casing Leakage in Offshore Gas Well[D], Beijing: China University of Petroleum (Beijing), 2019. 